an effective immune response. In the absence of co-stimulation, activation through the TCR can lead to a state of T-cell non-responsiveness called anergy, or to the related state of exhaustion. Prolonged activation of the TCR without co-stimulation can lead the $T$ cell to undergo a form of programmed cell death called apoptosis.

The addition of a co-stimulatory signal such as CD28 is notable because this signal has also been incorporated into another type of immunotherapy called chimaeric-antigen receptor $\mathrm{T}$ cell (CAR-T) therapy ${ }^{5}$, in which a receptor is engineered to both recognize a cancer-cell antigen and include T-cell activation domains such as CD3 and CD28. The main reason for including a CD28-binding domain in the trispecific antibody is T-cell co-stimulation. However, CD28 is also frequently expressed by multiple myeloma cells, so this might increase the antibody's affinity for the myeloma cells, and thus enable it to bind to cells in which CD38 is low, absent or masked by previous daratumumab therapy.

To confirm that the CD28-binding domain augmented the trispecific antibody's activity, the authors made versions of the antibody in which different combinations of the three binding domains were mutated. They tested these versions in 'humanized' model mice, which had human T cells and human myeloma cells. A functional CD28-targeting domain boosted T-cell activation above that observed using antibodies lacking this domain. This augmented $\mathrm{T}$-cell activation drove $\mathrm{T}$-cell proliferation and the expression of the anti-apoptotic protein $\mathrm{Bcl}-\mathrm{xL}$ in $\mathrm{T}$ cells, supporting the authors' hypothesis that having a co-stimulatory signal would prevent $\mathrm{T}$-cell apoptosis. The presence of the CD28-targeting domain on the antibody boosted the ability of $T$ cells to kill different myeloma cell lines in vitro and in the humanized mouse model, even at the lowest antibody dose tested.

The main limitation of this study is that the risk of a side effect called cytokine release syndrome (CRS), which can occur if the immune system is highly stimulated, is unknown. In CRS, the simultaneous activation of many $T$ cells causes excessive release of signalling molecules called cytokines from cells of the immune system, which drives inflammation. CRS can occur with bispecific antibodies and with CAR-T. It typically manifests as fever, but can progress to fatal multi-organ failure in severe cases ${ }^{6}$.

The authors report cytokine-related toxicities with their trispecific antibody when administered to monkeys by intravenous injection, but toxicity was less if it was delivered under the skin (subcutaneously) instead, leading to a more gradual exposure to the antibody. It is reassuring that the inclusion of the CD28-targeting domain did not lead to overwhelming CRS in these tests. However, a key caveat is that the amount of CD38 in monkeys is much less than in people with multiple myeloma, and the higher amount of CD38, and thus of antibody-mediated T-cell activation, would probably increase the risk of CRS in humans. But in terms of possible negative effects of the antibody on healthy non-cancerous cells, it is reassuring that only transient decreases in the number of normal white blood cells that express CD38, such as lymphocytes and myeloid cells, were observed

\section{“Targeting cancer using a trispecific antibody is an important conceptual advance."}

in monkeys treated with the antibody. Another limitation of the study is that the authors did not assess whether this trispecific antibody format might trigger an immune response against the antibody and cause its rapid destruction.

Targeting cancer using a trispecific antibody is an important conceptual advance, building on previous work by this group ${ }^{7}$ on a trispecific antibody that targets HIV. For multiple myeloma, fresh therapeutic approaches are needed, because even the most potent emerging therapies, including a CAR-T that targets an antigen called BCMA, are only temporarily effective for most people ${ }^{8-10}$. A trispecific antibody is a flexible platform that might offer a way to deliver precise combinations of immunomodulatory signals (for example, a co-stimulatory signal and a checkpoint blocker) specifically in the tumour microenvironment, which might be safer and more effective than the systemic administration of combinations of individual, single-specificity immunomodulatory antibodies. Such efforts to make immunotherapy more precise and potent than it is at present might be necessary to broaden the reach of immunotherapy to include the many types of cancer that have so far proved difficult to target.

Alfred L. Garfall and Carl H. June are in the Perelman School of Medicine, University of Pennsylvania, Philadelphia, Pennsylvania 19104, USA.

e-mail: cjune@upenn.edu

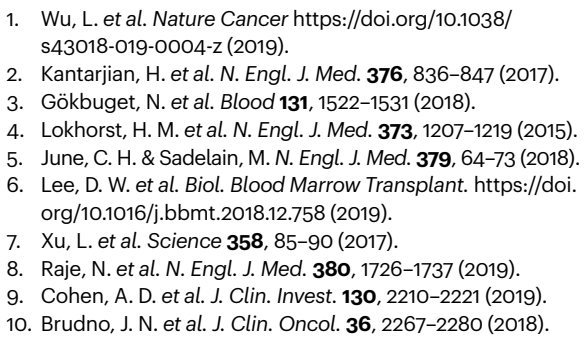

\title{
Microbial clues to a liver disease
}

\section{Martha R. J. Clokie}

Treatment options are limited for alcoholic hepatitis, a liver disease associated with high alcohol intake. Studies in mice reveal that the microorganisms responsible for this condition can be tackled by a viral treatment. See p.505

In 1984, the microbiologist Barry Marshall notoriously used himself as an experimental subject for his research, and drank the contents of a flask containing the bacterium Helicobacter pylori as part of his efforts to demonstrate that bacteria cause stomach ulcers'. On page 505, Duan et al. ${ }^{2}$ do not report taking such drastic action to investigate a bacterial connection to disease. Nevertheless, their careful analysis of a liver disease called alcoholic hepatitis, in studies of mice and analysis of samples from people who have the disease, also provide attention-grabbing evidence for the involvement of a suspected bacterial culprit.
Alcoholic hepatitis is a poorly understood condition related to high alcohol intake, and is difficult to treat. Previous experiments in mice have hinted that the gut-dwelling bacterium Enterococcusfaecalis might be involved ${ }^{3}$. However, E. faecalis is usually thought of as an old friend that inhabits the guts of many animals across the evolutionary tree, from humans to nematode worms ${ }^{4}$. This species usually represents less than $0.1 \%$ of all the bacteria in faecal samples from healthy people ${ }^{5}$. However, after antibiotic treatment, bacteria of the genus Enterococcus increase in prevalence to become one of the most common types of microbe in the gut ${ }^{6}$. E. faecalis can infect the 
blood, heart, bladder and brain, and teeth that have undergone root-canal surgery ${ }^{7,8}$.

Duan and colleagues analysed human faecal samples. They identified $E$.faecalis in the stools of about $80 \%$ of people with alcoholic hepatitis that they tested, and about $30 \%$ of the strains of $E$. faecalis present had genes that encode a toxin called cytolysin. Furthermore, people with the disease had almost 3,000 times more E.faecalis in their stool samples than did people who did not have alcoholic hepatitis. That isn't concrete proof that the disease is caused by this bacterium. However, the authors' data also show that the presence of cytolysin in stools correlates with mortality $-89 \%$ of the people whose faecal samples contained cytolysin died within 180 days of hospitalization, compared with only $3.8 \%$ of the people who had alcoholic hepatitis but whose stool samples lacked the toxin.

The authors next examined the connection between $E$. faecalis and liver disease in mice. The animals were colonized with strains of E. faecalis that either did or didn't make cytolysin, and some were then fed a high-alcohol diet, with others given an alcohol-free diet. Only the mice on the high-alcohol diet and that had been colonized with cytolysin-producing $E$. faecalis developed liver damage (Fig. 1a).

Then, using germ-free mice (which had no natural microorganisms), the authors transplanted stool samples from people with alcoholic hepatitis that contained $E$. faecalis strains in which cytolysin was either present or absent. Mice on a high-alcohol diet that were colonized with stools containing cytolysin displayed a range of signs indicating liver damage and the death of liver cells, whereas animals on such a diet and colonized with stools lacking cytolysin showed no major signs of liver damage.

To understand the disease-causing mechanisms, the authors isolated liver cells from the animals, and found that cell death in response to cytolysin exposure was dose-dependent. The response to cytolysin was the same whether or not the mice had received a high-alcohol diet. This suggests that, rather than alcohol causing alcoholic hepatitis by damaging the liver cells, damage arises because alcohol increases the permeability of the gut lining to allow cytolysin-producing $E$. faecalis to reach the liver and cause disease symptoms (Fig. 1a).

Given the limited treatment options for alcoholic hepatitis, the authors investigated whether steps might be taken to develop a therapy that exploits bacterium-targeting viruses called bacteriophages, or phages for short. Phages have the advantage over antibiotics of being highly specific, and so avoid also killing beneficial bacteria. Furthermore, because the surface of a human cell differs substantially from that of a bacterial cell,
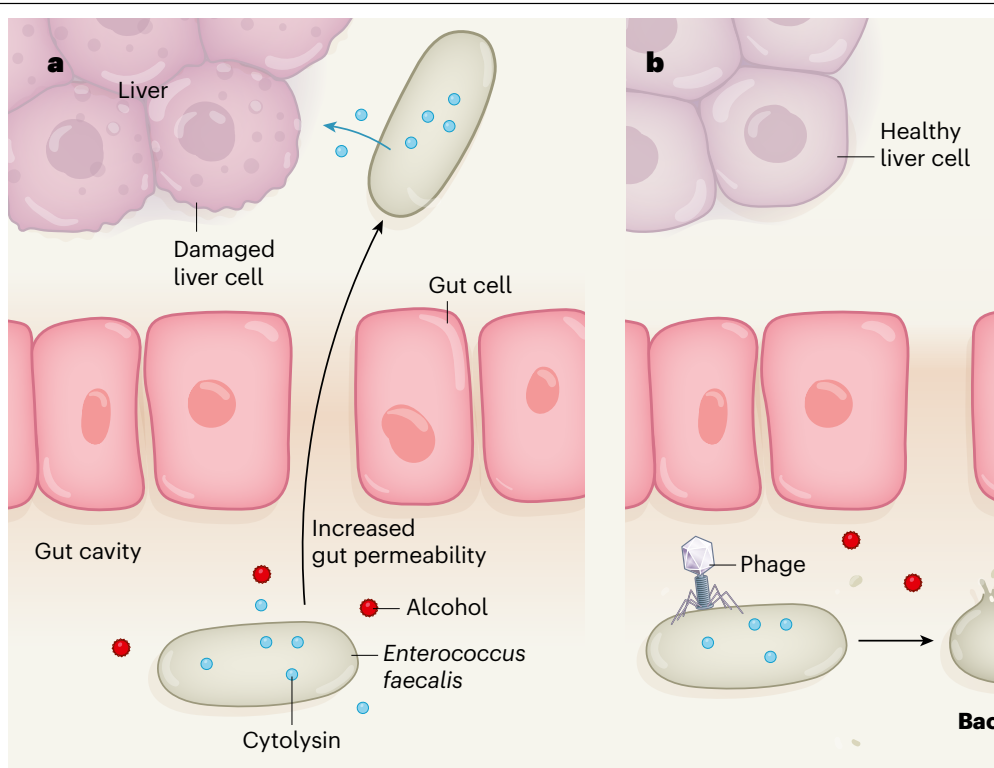

Figure 1 | Alcoholic hepatitis. Duan et al. ${ }^{2}$ report studies in mice of a liver disease called alcoholic hepatitis, and analysis of faecal samples from people who have the disease. a, The authors report that alcoholic hepatitis is associated with the presence of a strain of the gut-dwelling bacterium Enterococcusfaecalis that makes a toxin called cytolysin. These bacteria damage or kill liver cells, and the authors suggest that a high-alcohol diet increases gut permeability, thereby enabling the bacteria to move from the gut to the liver. b, To investigate possible new treatments for the disease, the authors explored the use of bacterium-targeting viruses called phages that specifically act on cytolysin-producing E.faecalis. When treated with these phages, E. faecalis-infected mice given a high-alcohol diet did not develop liver disease.

phages aren't thought to infect animal or human cells 9 .

Phages have been used to remove Salmonella and Shigella bacteria from infected human intestines for almost 100 years ${ }^{10}$. They have also been used to remove the disease-causing bacterium Clostridium difficile from artificial intestines, and from hamstersinfected with this bacterium $^{11,12}$. It has been suggested that they might one day be used in humans or animals to remodel the composition of the commu-

\section{"This study demonstrates the advantages of using phages in detective work."}

nity of gut microorganisms (the microbiota), to produce a healthier microbiota consisting of more bacteria associated with good health and fewer associated with disease ${ }^{13}$. The potential of $E$. faecalis-targeting phages to tackle human diseases is already being discussed ${ }^{7}$, and phages can kill antibiotic-resistant strains of $E$. faecalis associated with human bone and wound infections ${ }^{14,15}$ and dental cavities ${ }^{16}$. Furthermore, phages are being developed for use in the food industry to remove $E$. faecalis from cheese cultures to prevent the production of toxic waste products ${ }^{17}$.

To test whether a method could be developed to specifically remove cytolysin-producing $E$. faecalis from mice, the authors identified some phages that target these bacteria (Fig.1b) but leave other gut bacteria unaffected. Mice that received human stool samples and a high-alcohol diet and that were given $E$.faecalis-targeting phages had less liver damage than did mice given phages that killed a different bacterium not usually found in animals.

This study demonstrates the advantages of using phages in detective work to investigate the contributions of microbes to disease. The authors show that phages can be used to identify disease-causing bacterial components, and also raise the possibility that phages might offer potential treatment options. Further tests, including clinical trials, would be required to assess whether a phage approach would be useful in a human context. For example, phage treatment might help to target $E$. faecalis in the gut before a person receives a liver transplant.

In Duan and colleagues' study, the phages could treat a disease in which a causal component is a bacterium that normally resides in the gut, even though the disease site is elsewhere in the body. Although much phage research focuses on the use of these viruses to treat diseases associated with antibiotic-resistant bacteria, the work by Duan et al. raises the possibility of a much wider clinical role for them. There is growing evidence that gut microbes can affect the function of certain cells in the brain, and studies are ongoing to determine whether such microbes have a role in human brain diseases (see go.nature.com/2cp1kfk). Perhaps phages could become part of the next 
generation of targeted antimicrobial therapies for diseases that are currently difficult to treat. Indeed, there might be many diseases that we currently don't realize have a microbial component, and which could be tackled by phages.

Martha R. J. Clokie is in the Department of Genetics and Genome Biology, University of Leicester, Leicester LE1 7RH, UK. e-mail: mrjc1@leicester.ac.uk

1. Marshall, B. J., Armstrong, J. A., McGechie, D. B. \& Glancy, R. J. Med. J. Aust. 142, 436-439 (1985).

. Duan, Y. et al. Nature 575, 505-511 (2019).

3. Llorente, C. et al. Nature Commun. 8, 837 (2017)

4. Van Tyne, D. \& Gilmore, M. S. Annu. Rev. Microbiol. 68, 337-356 (2014).
5. Lebreton, F. et al. Cell 169, 849-861 (2017).

6. Ubeda, C. et al. J. Clin. Invest. 120, 4332-4341 (2010).

7. Bolocan, A. S. et al. Viruses 11, 366 (2019).

8. Arias, C. A. \& Murray, B. E. Nature Rev. Microbiol. 10 266-278 (2012).

9. Nguyen, S. et al. mBio 8, e01874-17 (2017).

10. Abedon, S. T., Kuhl, S. J., Blasdel, B. G. \& Kutter, E. M. Bacteriophage 1, 66-85 (2011).

11. Nale, J. Y., Chutia, M., Carr, P., Hickenbotham, P. T. \& Clokie, M. R. J. Front. Microbiol. 7, 1383 (2016).

12. Nale, J. Y., Redgwell, T. A., Millard, A. \& Clokie, M. R. J. Antibiotics 7, 13 (2018).

13. Whiteson, K. L. mSystems 3, 00166-17 (2018)

14. Melo, L. D. R., Ferreira, R., Costa, A. R., Oliveira, H. \& Azeredo, J. Sci. Rep. 9, 6643 (2019).

15. Barros, J. et al. Int. J. Antimicrob. Agents 54, 329-337 (2019).

16. Al-Zubidi, M. et al. Infect Immun. 87, e00512-19 (2019).

17. del Rio, B. et al. Front. Microbiol. 10, 566 (2019).

This article was published online on 13 November 2019

\section{Engineering}

\section{Virtual and augmented reality enhanced by touch}

\section{Xiao-ming Tao}

Conventional technologies for virtual and augmented reality simulate interactive experiences through visual and auditory stimuli. A technology that adds sensations of touch could find uses in areas from gaming to prosthetic feedback. See p.473

Human sensation includes the commonly known senses and less-recognized ones such as thirst, hunger and balance. Stimuli detected by sensory receptors are encoded into electrical signals that move along neural pathways to specific parts of the brain to be decoded into useful information. The whole process is complex. For instance, the sense of touch is a collection of several sensations, encompassing pressure, pain and temperature, and touch receptors are stimulated by a combination of mechanical, chemical and thermal energy. Until now, it has been a great challenge to incorporate sensations of touch into virtual and augmented reality. But on page 473 , Yu et al. report a skinintegrated technology that applies pressure, vibration or motion to the user, enabling communication between the user and a machine for virtual and augmented reality (X. Yu et al. Nature 575, 473-479; 2019).

The authors' technology consists of a soft, lightweight sheet of electronics that adheres to skin, and conforms to the body's shape, in a convenient, non-invasive and reversible manner (Fig. 1). The sheet contains arrays of vibratory actuators - mechanical components that convert electrical energy into vibrations. Each actuator comprises two connected parts: a coil of copper wire sealed in an acrylic base, and a permanent magnet mounted on a polymer beam. When an electric current passes through the coil, the magnet vibrates at the same frequency as that of the current.

Each actuator has a mass of only 1.4 grams and is millimetre-sized (12-18 $\mathrm{mm}$ in diameter
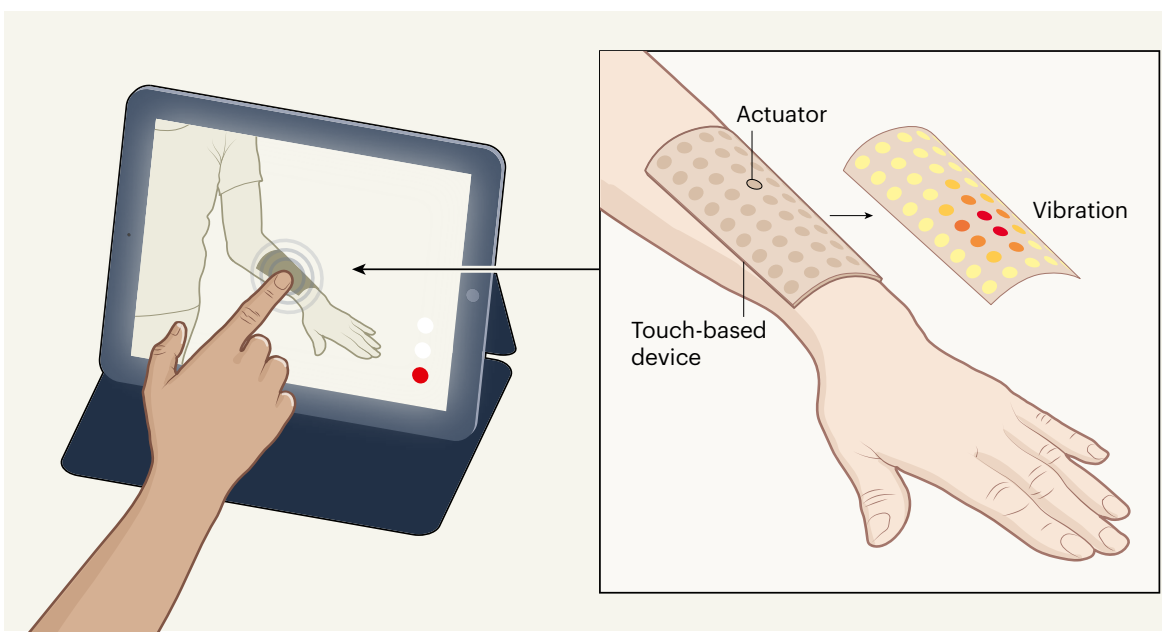

Figure 1 | Sense of virtual touch. Yu et al. present a device for incorporating touch-based sensations in virtual and augmented reality. The device consists of a lightweight sheet of electronics that softly laminates onto the skin. In this simple example, a touch screen displays a video feed of a person wearing the device, and a second person touches the image of the device on the screen. Mechanical components called vibrational actuators apply vibrations to the skin of the person wearing the device, providing a sense of virtual touch. The colours of the actuators represent their degree of activation from low (yellow) to high (red). 